\title{
„In the Doing of Hair, One Does Race“. Afroamerikanische Hairstyles als Technologien des Selbst
}

\author{
SILKE HACKENESCH
}

„In the beginning every body's hair was straight, but (...) the Negro's hair got bad when he went to Africa in consequence of the scorching sun and the hard rains of that climate“, lautete der mehr als fragwürdige Werbetext für ein hair straightening-Produkt, das 1889 in der Cleveland Gazette beworben wurde. Das angepriesene Produkt versprach, „bad hair good, short hair long, harsh hair soft and curly crisped hair straight" $\mathrm{zu}$ machen. ${ }^{1}$ In derselben Ausgabe der Gazette verurteilte ein anonymer Autor derartige Haarpflegemittel und riet seinen afroamerikanischen MitbürgerInnen, diesen „Humbug“ zu ignorieren und ebensolche Kosmetika zu boykottieren. Er nahm damit einen Standpunkt ein, der erst ein gutes halbes Jahrhundert später zum politischen Credo der Black PowerBe-wegung werden sollte, nachdem Black Panther Stokely Carmichael erklärt hatte: „We have to stop being ashamed of being black. A broad nose, a thick lip and nappy hair is us and we are going to call that beautiful whether they like it or not". ${ }^{2}$

Das Beispiel illustriert, wie kontrovers afroamerikanische Körperpraktiken, in diesem Falle Hairstyles, diskutiert worden sind, und dass dabei auch immer Auffassungen von race, gender und

Cleveland Gazette, 07.09.1889.

2 Zitiert nach William L. Van Deburg: New Day in Babylon. The Black Power Movement and American Culture, 1965-1975. Chicago, IL: University of Chicago Press 1992, S. 1992, 197f. In den afroamerikanischen Communities wird relativ glattes Haar, sei es von sich aus glatt oder sei es chemisch geglättet, häufig als gutes Haar, also good hair bezeichnet, wohingegen sehr „krauses“, stark gelocktes Haar als bad hair charakterisiert wird. Diese Bewertungen haben sich im Laufe der Zeit zu meist nicht hinterfragten Fakten/„Wahrheiten“ etabliert. Bezeichnungen wie nappy oder kinky beziehen sich ebenfalls auf nicht-geglättetes, stark "gekraustes“ Haar. Von einigen werden die letzten Zuschreibungen als herabwürdigend empfunden, für andere wiederum ist es eine affirmative Beschreibung ihrer Haartextur. 
class die Auseinandersetzungen beeinflussten und die Diskurse um Schwarzes Haar prägten. ${ }^{3}$ Mit einem Fokus auf dem 20. Jahrhundert soll in dieser Analyse beispielhaft dargestellt werden, welche Diskurse sich durch die Geschichte afroamerikanischen Haares zogen und in welchen Technologien des Selbst die diskursiven Effekte mündeten. Schnell wird dabei deutlich, dass sich die Debatten auf die Frage konzentrieren, ob Schwarzes Haar chemisch geglättet, also straightened, oder „natürlich“ getragen werden sollte, wobei der Umgang mit dieser Frage komplex, vielschichtig und gegensätzlich verläuft.

Afroamerikanische Körperpraktiken haben in der amerikanischen Kultur stets einen hohen Stellenwert eingenommen; die Wichtigkeit von Haarfrisuren wurde dabei bisher nicht hinreichend in der Forschung gewürdigt. Doch gerade die Geschichte der afroamerikanischen Haarkultur im 20. Jahrhundert macht deutlich, dass Schwarzes Haar als ein Schauplatz zum Teil heftiger Auseinandersetzungen und Aushandlungen fungiert. Susan Bordo hat gezeigt dass der Körper „is a battleground whose self-determination has to be fought for. The metaphor of the body as a battleground, rather than postmodern playground, captures, as well, the practical difficulties involved in the political struggle to empower ,difference““. ${ }^{4}$ Der Körper ist demnach ein Ort, an dem sich Diskurse der Disziplinierung und Normierung manifestieren; die Praktik des hair straightening (auch pressing genannt) ist dafür ein beredtes Beispiel. Hier artikuliert sich Macht ganz konkret als Diskurseffekt in einer alltäglichen Körperpraktik wie der des Haare Stylens. Die unterschiedlichen Haarpraktiken können mit Michel Foucault als Technologien des Selbst verstanden werden. Das Haar, beziehungsweise der Körper stellen jedoch nicht nur einen umkämpften Raum

3 Schwarz und weiß weisen in diesem Zusammenhang nicht auf die Farbe des Haares hin, sondern auf dessen Textur, meinen also Haar afroamerikanischer, bzw. euroamerikanischer Menschen. In den weiteren Ausführungen wird darauf verzichtet, die Zuschreibungen Schwarz und weiß in Anführungszeichen zu setzen, obwohl die Konstrukthaftigkeit dieser Variablen dem Text zugrunde liegt. Schwarz und weiß beschreiben keine biologischen Tatsachen, die die deutsche Übersetzung von Schwarz-, beziehungsweise weiß-sein zu suggerieren scheint; dennoch soll vermittelt werden, dass es sich trotz allem um wirkungsmächtige, sozial reale und fassbare Hierarchisierungsinstrumente handelt. Insbesondere Schwarz wird hier nicht mit einer naturalisierenden, rassistischen Konnotation begriffen, sondern im Sinne einer Reartikulation/Resignifikanz eines solidarischen, affirmativen politischen Begriffes, wohingegen weiß weder eine politische Identifikation, noch eine affirmative Selbstbezeichnung darstellt.

4 Susan Bordo: Unbearable Weight. Feminism, Western Culture, and the Body. Berkeley, CA: University of California Press 2003, S. 24. 
dar, sondern sind ihrerseits auch die Effekte verschiedenster Diskurse und performativer Praktiken, die sich in den Körper einschreiben. ${ }^{5}$ Variablen wie race und gender schreiben sich gleichwohl nicht nur ein, sondern produzieren einen Körper, der den normalisierenden Maßnahmen dieser Variablen unterworfen ist. Diesen Normalisierungsprozessen und dieser versuchten Unterwerfung widersetzen sich AfroamerikanerInnen - deren Körper in der amerikanischen Geschichte stets ein besonders prekärer Raum war - auch durch verschiedene Haarpraktiken, auf die in der folgenden Untersuchung eingegangen werden soll.

Hierfür ist das von Foucault in seinen späteren Arbeiten entwickelte Subjekt-Konzept fruchtbar, welches sich auf ein vormodernes Selbst bezieht. Foucault denkt, quasi als Aufkündigung des Poststrukturalismus, ein politisch aktives Subjekt, das mit einer bestimmten Form, mit einem bestimmten Maß an Freiheit ausgestattet ist. Diese Form der Freiheit ist die Bedingung für die Möglichkeit des Widerstandes. ${ }^{6}$ Sie bedeutet nicht, dass das Subjekt nicht mehr bestimmten Macht/Wissens-Komplexen unterworfen ist. Allerdings kann es sich zu den Formen seiner Unterwerfung in bestimmter Art und Weise von sich aus verhalten; das Subjekt kann sich fügen, oder sich widersetzen und somit den Status quo herausfordern. Durch eine Ethik der Sorge um sich selbst wird dem Subjekt so die Möglichkeit gegeben, der Unterwerfung durch die Moderne zu entkommen. Diese Überlegungen sind besonders hilfreich, um sich dem hier vorliegenden Untersuchungsgegenstand zu nähern. Laut Foucault hat „Kultur“ einen direkten Zugriff auf unsere Körper und auf die verschiedenen Körperpraktiken und körperlichen Gewohnheiten, denen wir tagtäglich nachgehen. Diese Praktiken, beziehungsweise Technologien wie das Stylen der Haare, sind eingebettet in verschiedene Diskurse. Technologien des Selbst ermöglichen es dem Einzelnen „aus eigener Kraft oder mit Hilfe anderer eine Reihe von Operationen an seinem Körper oder seiner Seele, seinem Denken, seinem Verhalten und seiner Existenzweise vorzunehmen, mit dem Ziel, sich so zu verändern, dass er einen gewissen Zustand des Glücks, der Reinheit, der Weisheit, der Vollkommenheit oder der Unsterblichkeit erlangt“. ${ }^{7}$ Es sind diese Selbstpraktiken, durch die wir uns individuell und sozial als Subjekte konstituieren. In diesem

5 Vgl. Judith Butler: Bodies that Matter. On the Discursive Limits of "Sex." London, New York: Routledge 1993, S. 9.

6 Vgl. Michel Foucault: "Die Ethik der Sorge um sich als Praxis der Freiheit" (1984), in: Ebd., Ästhetik der Existenz. Schriften zur Lebenskunst. Frankfurt/Main: Suhrkamp Verlag 2007, S. $266 f$.

7 Michel Foucault: „Technologien des Selbst“ (1982), in: Ebd., Ästhetik der Existenz. Schriften zur Lebenskunst. Frankfurt/Main: Suhrkamp Verlag 2007, S. 289. 
Sinne ist das künstliche/chemische Glätten der Haare eine Technologie des Selbst und das „natürliche“ Wachsen des Haares, beziehungsweise das Tragen eines Afros, kann als eine Gegentechnologie gelesen werden, da es im offenen Widerspruch zu dominanten Diskursen steht und als Körperpraktik mehrheitlich sozial geächtet ist. Es stellt darüber hinaus eine Befreiung von ethischen Konventionen und eine subversive ästhetische Inszenie-rung des Subjekts dar. Die Praxis des straightening hingegen ist gesellschaftlich akzeptiert und sogar erwünscht. Der Diskurs um das straightening ist daher der dichteste und mächtigste; er produziert den höchsten Wahrheitsgehalt.

Schwarzes Haar operiert als racial signifier; es ist sofort sichtbar, jedoch im Gegensatz zur Hautfarbe leichter veränderbar: "Caught on the cusp between self and society, nature and culture, the malleability of hair makes it a sensitive area of expression". 8 Historisch betrachtet ist Schwarzes Haar stets ein Ort gewesen, an dem die soziale Position von Afroamerikanern verhandelt worden ist. An etlichen Frisuren, seien es straightened/geglättete Haarfrisuren, der Conk, der Afro, Cornrows, Braids oder Dredlocks, entzündeten sich Debatten, in denen es häufig nicht in erster Linie um Attraktivität, sondern um Respektabilität, Fortschritt, das Einreißen von gesellschaftlichen Konventionen und Geschlechter-normen, und das Demonstrieren von politischen Überzeugungen ging.

\section{"Should Negro Women Straighten their Hair?“}

Bereits im frühen 19. Jahrhundert warben von Euroamerikanern geführte Unternehmen mit Produkten, die das Haar von Afroamerikanerinnen glatt und somit vermeintlich schöner machen sollten. ${ }^{9}$ In ihrem Buch Hair Raising problematisiert Noliwe Rooks diese Art der Bewerbung, indem sie darauf hinweist, dass das propagierte Schönheitsideal für Schwarze Frauen mehrheitlich unerreichbar war. Infolge dessen griffen diese zu drastischen Methoden, um good hair zu bekommen und den zuverlässigsten Indikator für afrikanische Herkunft und vermeintliche „Inferiorität“ los zu werden, nämlich nappy/kinky hair. Die Verknüpfung von rassistischer Ideologie mit einem europäischen Schönheitsideal war besonders problematisch für Schwarze Frauen, da sie von ebenjenem Ideal auf mehre-

8 Kobena Mercer: Welcome to the Jungle. New Positions in Black Cultural Studies. London, New York: Routledge 1994, S. 103.

9 Vgl. Maxine Craig: Ain't I a Beauty Queen? Black Women, Beauty, and the Politics of Race. Oxford, New York: Oxford University Press 2002, S. 26. 
ren Ebenen ausgeschlossen waren. ${ }^{10}$ Es wäre jedoch zu kurz gegriffen, Haarpraktiken einzig und allein im Kontext von Schönheitsstandards einer weißen Vorherrschaft zu analysieren, weil dabei unberücksichtigt bleiben würde, dass Afroamerikanerinnen eigene, distinkte Auffassungen von Attraktivität entwickelt haben. ${ }^{11} \mathrm{Mehr}$ noch als das Imitieren euroamerikanischer Schönheitsideale galt hair straightening im eigenen Selbstverständnis als gut-frisiert-sein und war eben auch ein Symbol für persönlichen Stolz, die eigene Wertschätzung sowie Respektabilität. ${ }^{12}$ Es ist für die Analyse wenig aufschlussreich, das Anpassen, beziehungsweise die Identifikation mit aktuellen Schönheitsstandards und sozialen Normen einzig mit einem (unbewussten) Wunsch nach weiß-sein zu interpretieren. In Skin Trade macht Ann DuCille deutlich: „The absence of black images in the ,social mirror leaves the black child with little other than white subjects for self-reflection and self-projection. But a child's dreaming in the color scheme privileged by the world around her is not necessarily the same as wanting to be that color". ${ }^{13}$ Sich Schönheitsvorstellungen anzueignen, beziehungsweise sich Styles zu Eigen zu machen, die wei $\beta$ codiert sind, muss demnach nicht zwangsläufig implizieren, dass das Subjekt tatsächlich bevorzugt weiß wäre.

$\mathrm{Zu}$ Beginn des 20. Jahrhunderts etablierte sich, auch als Konsequenz aus der praktizierten Segregation in den USA, eine eigenständige afroamerikanische Schönheitskultur und -industrie, die sich als äußerst lukrative ökonomische Nische erwies. Weil Schwarze Frauen ebenso wie Weiße auf ihr äußeres Erscheinungsbild bedacht waren und in erster Linie modern sein wollten, was untrennbar mit Körperpraktiken wie Schminken und Frisieren verknüpft war, schossen „Schönheitsschulen“ aus dem Boden, in denen sich die Frauen zu Friseurinnen und Kosmetikerinnen ausbilden lassen konnten. ${ }^{14} \mathrm{Ab}$ dem Zeitpunkt, ab dem sich die Schönheitsindustrie explizit an Afroamerikanerinnen wandte und massiv Reklame für hair straightening-Produkte machte, wurde diese Haarpraxis jedoch immer mehr zu einem Streitpunkt, der innerhalb der afroamerika-

10 Noliwe M. Rooks: Hair Raising. Beauty, Culture, and African American Women. Piscataway, NJ: Rutgers University Press 1996.

11 Vgl. Tracy O. Patton: „Hey Girl, Am I More than my Hair? African American Women and their Struggles with Beauty, Body Image and Hair", in: National Women's Studies Journal 18 (2006), S. 24-51, hier S. 27.

12 Vgl. M. Craig: Ain't I a Beauty Queen?, S. 15.

13 Ann DuCille: Skin Trade. Cambridge, MA: Harvard University Press 1996, S. 13 (Hervorhebung im Original).

14 Vgl. Julia K. Blackwelder: Styling Jim Crow. African American Beauty Training During Segregation. College Station, TX: Texas A\&M University Press 2003, S. 6. 
nischen Communities vor dem Hintergrund eines Black PrideDiskurses kontrovers diskutiert worden ist. Diese Debatten, die im Folgenden skizziert werden, sollten allerdings nicht über die Tatsache hinweg täuschen, dass chemisch behandeltes und dadurch geglättetes Haar mit Abstand der populärste Style unter afroamerikanischen Frauen während des gesamten 20. Jahrhunderts gewesen ist.

Anhand von afroamerikanischen Frauenzeitschriften und Anzeigen für Kosmetikprodukte aus der ersten Hälfte des 20. Jahrhunderts lässt sich ablesen, wie sehr Auffassungen von race mit denen von euroamerikanisch dominierten Schönheitsidealen und Modevorstellungen verknüpft waren. Die nationale amerikanische Kultur war gesättigt mit Bildern, die unter anderem langes, glattes (bone straight) Haar als essentiellen Bestandteil von Femininität erachtete. Damit wurde das Aussehen von Schwarzen Frauen ohne, dass es explizit hätte genannt werden müssen, als weniger attraktiv konstruiert. Diese euroamerikanischen Schönheitsstandards waren auf das Engste verknüpft mit sozialer Mobilität und Auffassungen von Klassenzugehörigkeit. Da geglättetes Haar mit vorherrschenden gesellschaftlichen Normen übereinstimmte, war die Praktik des straightening „a matter of economic, social, and political survival“.15 Höhere Attraktivität, die durch das hair straightening erreicht würde, führe somit zugleich zu ökonomischen Erfolg. Der Text einer Anzeige für ein hair straightening-Produkt in der afroamerikanischen Zeitung St. Louis Palladium aus dem Jahre 1905 lautete entsprechend: „You owe it to yourself, as well as others who are interested in you, to make yourself as attractive as possible. Attractiveness will contribute much to your success - both socially and commercially. Positively nothing detracts from your appearance as short, matted, unattractive curly hair“. ${ }^{16}$

Die Annahme, dass das Aussehen ein entscheidender Faktor im persönlichen, sozialen und beruflichen Vorankommen sei, erinnert an die Politik Booker T. Washingtons, der seinen Studierenden am Tuskegee Institute in Tuskegee, Alabama, „the basics of Victorian standards of public conduct and personal appearance“, nahe brachte, was „being well groomed“ mit einschloss. ${ }^{17}$ Maxine Craig, Autorin von Ain't I a Beauty Queen? formuliert es folgendermaßen: „A woman who put time and money into her appearance was dignified,

15 Mariame Kaba: „When Black Hair Tangles With White Power“, in: Juliette Harris/Pamela Johnson (Hg.), Tenderheaded. A Comb-Bending Collection of Hair Stories. New York: Pocket Books 2001, S. 106.

16 Zitiert nach N. Rooks: Hair Raising, S. 33.

17 Bruce M. Tyler: „Black Hairstyles. Cultural and Socio-Political Implications“, in: The Western Journal of Black Studies 14 (1990), S. 235-250, hier S. 237. 
and her dignity spoke well of the race“.18 Hair straightening war eine von der Mehrheit der Frauen ausgeübte Körperpraktik, relativ unabhängig von ihren ökonomischen Möglichkeiten. Reichte das Geld nicht aus, um einen Schönheitssalon zu besuchen, oder wohnten die Frauen in so ländlichen Gegenden, dass es keinen beauty parlor in der näheren Umgebung gab, wurde nicht selten die Küche einer Nachbarin in einen Friseursalon umgewandelt, in dem man sich traf und gegenseitig das Haar machte. Henry Louis Gates Jr. Beschreibt eine häufige Szene aus seiner Kindheit, in der seine Mutter ihren Freundinnen die Haare frisierte. Dafür wurde kurzerhand die Küche umfunktioniert, weil das Glätteisen auf dem Herd erwärmt werden musste, mit dem die geölten Haare geglättet werden konnten: „I liked what that smell meant for the shape of my day. There was an intimate warmth in the women's tones as they talked with my mama while she did their hair“. ${ }^{19}$

Auch wenn sehr viele AfroamerikanerInnen von den dominanten, weißen Auffassungen darüber, was als schön und attraktiv galt, beeinflusst waren, so gab es dennoch Stimmen -vorwiegend der Schwarzen männlichen Elite-, die ein Schönheitsideal forderten, das dem afrikanischen Aussehen Rechnung trug, und sich vorherrschenden Schönheitsstandards widersetzen sollte. Im frühen 20. Jahrhundert fokussierten die Diskurse um Schwarzen Stolz in der Tat auf das Verlangen nach neuen, eigenen Bildern von Schwarzer Schönheit, die die schädigenden Repräsentationen der dominanten euroamerikanischen Kultur herausfordern und konterkarieren sollten. Einer der prominentesten Vertreter eines positiven Schwarzen Selbstverständnis und pan-afrikanischer Einheit im frühen 20. Jahrhundert war Marcus Garvey, der die Praktik des straightening vehement verurteilte. Für ihn war sie Ausdruck einer Negation des eigenen Schwarz-seins. ${ }^{20}$ Ähnlich argumentierte ein anonymer Schreiber 1920 im Half-Century Magazine -herausgegeben von einer Afroamerikanerin und adressiert an „the masses across class“- der in einem Brief mit dem unmissverständlichen Titel „Betrayers of the Race“ ebenfalls die Ansicht vertrat, dass Afroamerikanerinnen den Betrügereien euroamerikanischer Schönheitsfirmen aufsitzen würden. ${ }^{21}$ Ebenjene „Betrogenen“ rechtfertigten sich, sie gingen viel-

18 M. Craig: Ain't I a Beauty Queen?, S. 35.

19 Henry Louis Gates, Jr.: „It all Comes Down to the Kitchen“, in: Juliette Harris/Pamela Johnson (Hg.), Tenderheaded. A Comb-Bending Collection of Hair Stories. New York: Pocket Books 2001, S. 24.

20 Vgl. Obiagele Lake: Blue Veins and Kinky Hair. Naming and Color Consciousness in African America. Westport, CT: Praeger 2003, S. 68.

21 Vgl. Noliwe M. Rooks: Ladies' Pages. African American Women's Magazines and the Culture that made them. New Brunswick, NJ: Rutgers University Press 2004, S. 69. 
mehr mit der (euroamerikanisch dominierten) Mode und würden ihr Äußeres zelebrieren, anstatt sich dessen zu schämen und es drastisch verändern $\mathrm{zu}$ wollen. ${ }^{22}$ Tatsächlich wurde die Formel hair straightening gleich weiß-sein von Afroamerikanerinnen, die in der Schönheitsindustrie ein Vermögen machten und Produkte wie hair straightener und skin bleaching cremes (Cremes, die mit häufig aggressiven Wirkstoffen die Haut vermeintlich aufhellen) bewarben, tunlichst vermieden. Sie betonten indes, dass ihre Produkte das Haar pflegten, es besser kämmbar machten und Haarausfall vorbeugten, beziehungsweise dass die Cremes das Hautbild verbesserten.

\section{„The Greatest Benefactress of our Race“}

Eine der ersten Frauen in der afroamerikanischen Schönheitsindustrie war Sarah Bredlove Walker, besser bekannt unter dem Namen Madame C. J. Walker, die mit Hilfe ihrer Produkte die erste afroamerikanische self-made Millionärin in den USA wurde und eine prominente Rolle in den Schwarzen Communities einnahm. Sie war Mitglied der National Association for the Advancement of Colored People (NAACP), die sie finanziell großzügig unterstützte, und sie kämpfte öffentlich gegen Lynchings und die Diskriminierung von AfroamerikanerInnen. Sie sah in der Anwendung ihrer Produkte keinen Ausdruck von einem negativen internalisierten Selbstbild, sondern betonte im Gegenteil, dass ihre Kosmetik die Attraktivität ihrer Kundinnen erhöhen würde, wodurch diese wiederum ihr Selbstwertgefühl steigern würden. Sie selbst betrachtete ihre Philanthropie und ihr gewinnträchtiges Kosmetikunternehmen durchaus als politisch, eine Wahrnehmung, die für viele Zeitgenossen schwer nachvollziehbar war, und sagte über sich selber, sie sei dem „racial uplift“ verschrieben. ${ }^{23}$ Während ihrer gesamten Karriere unterminierte sie ganz bewusst Ideologien über race, gender und sozialen Status, die in der weißen wie auch der Schwarzen Mittelschicht vorherrschten. Sie inszenierte sich selbst als Beispiel dafür, wie eine relativ ungebildete Schwarze Frau aus einfachsten Verhältnissen Erfolg haben konnte und forderte andere Schwarze Frauen auf, es ihr gleich zu tun, indem sie Vertreterinnen ihres Unternehmens wurden: „Women (who) saw her photo and heard her life story (...) clamored to take her course and sit for her treatments. The twin

22 Vgl. N. Rooks: Hair Raising, S. 41.

23 Vgl. das Werbeheftchen The Key to Beauty, Success, Happiness. Vertrieben durch die C. J. Walker Manufacturing Co., Indianapolis, Indiana (Rare Book and Manuscript Division, Schomburg Center for Research in Black Culture, New York Public Library, New York). 
promises of enhanced beauty and financial gain - not to mention Madam Walker's own phenomenal personal example - served as a magnet to women who had always believed they would never be more than maids and laundresses"..24 Die Tatsache, dass Walker somit die Frauen aufforderte, der häuslichen Sphäre zu entfliehen und eigenes Geld zu verdienen, führte innerhalb der afroamerikanischen Communities einerseits zu Spannungen, andererseits aber auch zu einem Umdenken der Geschlechterrollen. Gerade Frauenmagazine, die sich an Afroamerikanerinnen richteten, griffen die Philosophie Walkers auf und kreierten einen Raum für eine neue Schwarze, weibliche Identität. Im Jahre 1917 erschien in dem Magazin Woman's Voice, dass sich an afroamerikanische Frauen der Arbeiterklasse richtete, ein Artikel, der seinen Leserinnen Ratschläge anbot, wie sie mit den Transformationen innerhalb ihrer Familien und Beziehungen umgehen könnten, sobald sie arbeiteten. ${ }^{25}$ Dass Afroameri-kanerinnen arbeiten mussten, war indes kein Novum. Aufgrund der häufig prekären ökonomischen Lage war es selten so, dass sich die Tätigkeiten Schwarzer Frauen auf den häuslichen Bereich beschränkten. Wo Euroamerikanerinnen für das Recht zu arbeiten kämpfen mussten, gehörte dies seit der Sklaverei zum Alltag von Afroamerikanerinnen. Aber der entscheidende Unterschied war, dass Frauen, wenn sie für das Unternehmen von Madame Walker arbeiteten oder einen eigenen Schönheitssalon eröffneten, nicht für EuroamerikanerInnen arbeiteten, sondern für andere Schwarze Frauen. Sie entzogen sich damit der etwaigen Kontrolle der Schwarzen Männer ebenso wie der Kontrolle der weißen Vorherrschaft, von der sie ökonomisch nicht mehr abhängig waren.

Das Schaffen von Madame Walker veranschaulicht, wie sehr im Diskurs um Schwarzes Haar nicht nur mitunter gegensätzliche Auffassungen von race verhandelt wurden, sondern ebenso unterschiedliche Verständnisse von Geschlechterkonventionen. Walkers Einsatz für die relative ökonomische Unabhängigkeit Schwarzer Frauen und ihr modernes Frauenbild brachten ihr viel Widerstand seitens der männlichen afroamerikanischen Mittelschicht ein. Bei der National Negro Business League Convention im Jahre 1912 weigerte sich Booker T. Washington, Walkers Präsenz anzu-erkennen. Auch wenn er argumentierte, er halte nichts von Walker, weil er dem Boom in der Kosmetikbranche skeptisch gegenüber stehe, kann man davon ausgehen, dass ihn ein nicht geringes Ma $\beta$ an Sexismus und Chauvinismus zu diesem Schritt bewogen hat. Dies verwundert umso mehr, als Walker mit Hilfe ihres eigenen Lebens und ihrer eigenen Karriere Entschlossenheit, Eigenständigkeit und

24 A'Lelia Bundles: On Her Own Ground. The Life and Times of Madam C. J. Walker. New York: Scribner 2001, S. 96.

25 Vgl. N. Rooks: Hair Raising, S. $97 f$. 
Schwarzen Stolz propagierte, alles im Einklang mit den politischen Botschaften eines Marcus Garvey oder eines Booker T. Washington. ${ }^{26}$ W.E.B. Du Bois verurteilte Garvey und Washington für ihre Kritik an Walker, die ihm simplifiziert erschien. Seiner Ansicht nach war eine Reduzierung von hair straightening auf eine Praktik zur Emulation von weiß-sein allzu sehr vereinfacht. Nach ihrem Tod im Jahre 1919 bezeichnete er Walker und den Erfolg ihres Unternehmens als epochemachend. ${ }^{27}$ Wie widersprüchlich die Kritik zum Teil war, wird auch aus der Tatsache deutlich, dass Garvey Walker und ihre Produkte in seiner Zeitung Negro World zwar aufs Schärfste verurteilte, dieselbe Zeitung jedoch zu einem Drittel aus Anzeigen für Aufhellungscremes und Glätteisen, beziehungsweise chemischen Lösungen, mit denen sich das Haar glätten lie $\beta$, bestand, aus denen sie sich hauptsächlich finanzierte. ${ }^{28}$ Auch wenn Zeitungen wie Negro World und Crisis Produkte ablehnten, die ein euroamerikanisches Schönheitsideal propagierten und stattdessen Stolz auf das eigene Schwarz-sein, sowie ein Schwarzes Bewusstsein schaffen wollten, so waren sie doch ökonomisch von diesen Anzeigen abhängig, da es die mit Abstand populärsten Produkte in der afroamerikanischen Bevölkerung waren. ${ }^{29}$ Während des gesamten 20. Jahrhunderts wurde die Vermarktung und Popularisierung von straightened hairstyles von afroamerikanischen Zeitungen und Magazinen unterstützt. Und auch wenn einige Führungspersönlichkeiten der Schwarzen Mittelschicht und politischen Elite über die moralischen und sozialen Implikationen dieser Praktik stritten, stand der Beliebtheit des Styles kaum etwas im Wege. Diese Entwicklung ging einher mit der zunehmenden Kommerzialisierung der Schönheits-

26 Vgl. T. Patton: „Hey Girl, Am I More than my Hair?“, S. $28 \mathrm{f}$.

27 Vgl. Mark Higbee: „The Hairdresser and the Scholar“, in: Juliette Harris/ Pamela Johnnson (Hg.), Tenderheaded. A Comb-Bending Collection of Hair Stories. New York: Pocket Books 2001, S. 11.

$28 \mathrm{Vgl}$. Ayana Byrd/Lori L. Tharps: Hair Story. Untangling the Roots of Black Hair in America. New York: St. Martin's Press 2001, S. 39. An dieser Stelle sei darauf hingewiesen, dass Walker sowohl mit Marcus Garvey als auch mit W.E.B. Du Bois politisch eng kooperierte. Sie empfing zum Beispiel Garvey in ihrer Villa in New York, um über die Gründung einer International League of Darker Peoples zu beraten (Vgl. David Levering Lewis: W.E.B. Du Bois. The Fight for Equality and the American Century, 1919-1963. New York: Henry Holt 2000, S. 58-60).

29 Vgl. den Folder „NAACP Crisis, General Operational File, Apex Hair co. Inc., 1946-50“, der Rechnungen enthält, die das Kosmetikunternehmen Apex von der Crisis für das Schalten von Anzeigen für Schönheitsprodukte erhalten hat, beziehungsweise Zahlungsbelege für die Anzeigen von Apex (Records of the NAACP, Part II: F: Crisis Files, 1940-55, F2: Apex Hair Co., 1941-49, Manuscript Reading Room, Library of Congress, Washington, D.C.). 
kultur in den 20er und 30er Jahren wie sie von Madame Walker und anderen vorangetrieben worden war. Die Schönheitsindustrie, und das spiegelt sich ebenfalls in der Prävalenz dieser Anzeigen wieder, war der zentrale Bereich, in dem Afroamerikanerinnen aktiv an der amerikanischen Konsumkultur partizipierten (Abb. 1). Nicht nur, dass sie trotz oft geringerer Einkommen verhältnismäßig mehr Geld für Kosmetika ausgaben, mehr noch, „beauty culture was one industry that flourished in black communities, and it was an industry dominated by black businesswomen“. ${ }^{30}$

\section{Printanzeige für einen Hair Straightener}

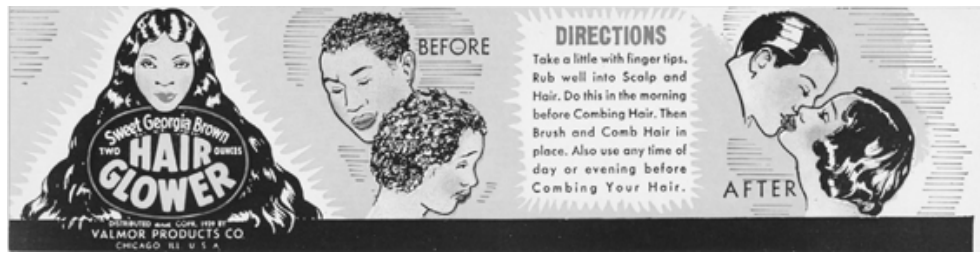

Abb. 1: Beispiel einer Printanzeige für das Haarpflegeprodukt Sweet Georgia Brown Hair Glower der Firma Valmor Products, mit dem die Haare geglättet werden sollten. Vorher/Nachher-Bilder waren eine häufig eingesetzte Strategie bei der Bewerbung von hair straighteners und skin bleaching creams. Nicht selten sind die in der Werbung abgebildeten Menschen nach der Anwendung des verheißungsvollen Produkts, wie in diesem konkreten Beispiel, nicht nur attraktiver (in der Logik der Reklame), sondern auch beruflich erfolgreicher, oder glücklich verliebt. (Sweet Georgia Brown Hair Glower, ca. 1934-1946)

Kosmetikstudios (beauty parlors) und Herrensalons (barber shops) bildeten einen weiteren Eckpfeiler in der Expansion einer afroamerikanischen Schönheitsindustrie. Kamen Kosmetikerinnen und Friseurinnen in den 1920er und 1930er Jahren noch zu ihren Kundinnen nach Hause oder betrieben kleine Salons in ihren Nachbarschaften, so entstanden in den späten 1940er und 1950er Jahren in allen großen Städten des Nordens Kosmetiksalons, die in erster Linie Kundinnen aus der Mittelschicht anzogen. ${ }^{31}$ Diese Schönheitssalons, waren es nun kleine Läden in der Nachbarschaft, große Salons in den Städten oder eine Küche, die für einen Tag in einen beauty parlor umfunktioniert wurde, waren von großer kommunaler

30 Susannah Walker: Style and Status. Selling Beauty to African American Women, 1920-1975. Lexington, KY: The University Press of Kentucky 2007, S. 7.

31 Vgl. S. Walker: Style and Status, S. $115 f$. 
Bedeutung für die Kundinnen und Kunden. In der amerikanischen Gesellschaft ist der Friseursalon, neben der Kirche, ein nach wie vor am stärksten segregierter, und somit intimer und vertrauenswürdiger Ort, in dem neben alläglichen Begebenheiten immer auch Tagespolitik und Auswirkungen von Rassismus verhandelt worden sind. ${ }^{32}$

Für Afroamerikanerinnen ist das straightening eine Körperpraktik gewesen, die das Haar handhabbarer machen und es somit erlauben sollte, zu experimentieren und mehrere Styles auszuprobieren. Glätten wurde hier als kulturelle Praktik verstanden, die es den Frauen erlaubte „slick, modern, and attractive“ auszusehen. ${ }^{33}$ Auf einer weiteren diskursiven Ebene wurden diese glatten, modernen Frisuren als respektables Äußeres gewertet, das sowohl einen Mittelschichtstatus kommunizierte, als auch einen Stolz auf das eigene Aussehen. Es ist auch gegenwärtig noch so, dass der Aspekt der Kreativität und der performative Akt des Stylens von zentraler Bedeutung sind. Gerade Afroamerikanerinnen der Arbeiterklasse glätten ihre Haare erst, um sie dann anschließend in äußerst elaborierten und kunstvoll drapierten Frisuren anzuordnen. ${ }^{34}$ In diesem Kontext, wie er auch von Vertreterinnen wie Madame Walker propagiert worden ist, steht das geglättete Haar nicht in einem eklatanten Widerspruch zum Afro, der ab den 1960er Jahren zu dem Style schlechthin erklärt worden ist, mit dem sich ein Schwarzes Bewusstsein, Schwarzer Stolz und Schwarzer Nationalismus auszudrücken hatte. Auch wenn der Afro nicht unter Verdacht stand, Respektabilität zu kommunizieren und zu ökonomischen Erfolg zu führen, so lässt sich dennoch argumentieren, dass beide Styles Selbstbewusstsein und Wertschätzung zum Ausdruck bringen sollten. Doch wie verhielt es sich mit Schwarzen Männern, die sich ihre Haare glätteten? Analysiert man den Kontext, in dem der so genannte Conk, wie man das chemisch geglättete Haar von afroamerikanischen Männern nannte, in den 40er und 50er Jahren popularisiert worden ist, so wird schnell deutlich, dass sich die diskursiven Effekte, die im conking mündeten, stark von denen unterscheiden, die bislang analysiert worden sind.

32 Vgl. Kimberly Battle-Walters: Sheila's Shop. Working-Class African American Women Talk About Life, Love, Race, and Hair. Lanham, MD: Rowman \& Littlefield 2004; bell hooks: „Straightening Our Hair“, in: Juliette Harris/ Pamela Johnson ( $\mathrm{Hg}$. ), Tenderheaded. A Comb-Bending Collection of Hair Stories. New York: Pocket Books 2001; J. Blackwelder: Styling Jim Crow.

33 Vgl. A. Byrd/L. Tharps: Hair Story, S. 40.

34 Vgl. Rose Weitz: „Women and their Hair. Seeking Power through Resistance and Accommodation“, in: Gender \& Society 15 (2001), S. 667-86, hier S. 667. 


\section{„See the Pimps with the Conk on their Hair“}

Als während der 1940er und 1950er Jahre die afroamerikanische Schönheitsindustrie regelrecht explodierte, gingen einige junge Afroamerikaner dazu über, ihre Haare nicht mehr ganz kurz zu tragen, sondern sie ebenfalls wachsen zu lassen und zu glätten. Für diesen Style, Conk genannt, wurde das Haar geglättet, um dann das dadurch verlängerte Haar wellenförmig nach hinten zu legen. Die Lauge, die häufig für das conking verwendet wurde, hellte das Haar auf und gab ihm einen rötlichen Farbstich. Das erste Produkt, das speziell für Männer auf den Markt gebracht wurde, hie $\beta K K K$, als Abkürzung für Knocks Kinks Krazy. Es blieb jedoch, obwohl an sich innovativ, unerfolgreich, da die meisten Kunden der Ironie des Namens wenig abgewinnen konnten. ${ }^{35}$ Die prominentesten Vertreter des Conk waren James Brown, der frühe Marvin Gaye und die spätere Galionsfigur der Black Power-Bewegung Malcolm X. In seiner Autobiographie berichtet Malcolm X von seiner Adoleszenz inmitten der Zoot Suiters in Harlem, die sich in erster Linie aus jungen afroamerikanischen und lateinamerikanischen Männern der unteren sozialen Schichten zusammensetzten. Da die Auto-biographie dazu intendiert war, seinen Aufstieg bei der Nation of Islam (NOI) nachzuzeichnen und seine nationalistische, panafrikanische Politik zu vermitteln, sollten diese Memoiren „as a literary construction, a cliché that obscures more than it reveals" gelesen werden; Robin D. G. Kelley bemerkt dazu: „The story is tragically dehistoricized“. ${ }^{36}$ In der nachträglichen Erzählung, in der er sich auch ausführlich mit seinem ersten Conk auseinander setzt, kann Malcolm X diesen nur noch als Akt der Erniedrigung und Degradierung wahrnehmen. Der Wunsch nach einem Conk ist für ihn Ausdruck des Verlangens, weiß-sein zu wollen und Beweis dafür, wie sehr er die Normen und Werte eines Systems internalisiert hatte, dass ihn sozial und politisch unterdrückte und für hässlich und deviant erklärte:

This was my first really big step toward self-degradation: when I endured all of that pain, literally burning my flesh to have it look like a white man's hair. I had joined that multitude of Negro men and women in America who are brainwashed into believing that the black people are ,inferior' -and white

35 Vgl. A. Byrd/L. Tharps: Hair Story, S. 43f.

36 Robin D. G. Kelley: Race Rebels. Culture, Politics, and the Black Working Class. New York: The Free Press 1994, S. 162. 
people ,superior'- that they will even violate and mutilate their God-created bodies to try to look , pretty" by white standards. ${ }^{37}$

Der Schmerz, den er bei der gesamten Prozedur des conking aushalten musste, ist ein zentraler Bestandteil seiner Erzählung und unterstreicht die Absurdität, die er seiner Handlung retrospektiv beimisst. ${ }^{38}$ In Race Rebels argumentiert Kelley überzeugend, dass Malcolm X den Conk aus seinem Kontext reißt, um die eigene Argumentation zu stützen, der Conk sei der ultimative Ausdruck von Selbsthass und Negierung des eigenen Schwarz-seins. Dies ist insofern problematisch, als dass der Conk auch als Gegentechnologie und körperliches Widerstandsmoment gelesen werden kann. Zum einen wurde der Conk in erster Linie von Zoot Suiters getragen, die sich kaum dem Verdacht aussetzten, sich weißen Normvorstellungen zu unterwerfen. Vielmehr war er für sie ein Mittel, sich dem stereotypen Bild des afroamerikanischen Migranten aus dem Süden zu erwehren und eine Möglichkeit, relative ökonomische Unabhängigkeit zu demonstrieren. Sie waren vielleicht nicht reich, aber sie hatten genug Geld, um sich einen Conk (auch process genannt, da das Haar processed/chemisch behandelt wurde) verpassen zu lassen. In einem Interview mit Maxine Craig formuliert es ein Mann so: „A process showed that I had money and prestige. I could afford to do anything I wanted. I was showing that I wasn't poor“.39 Der Conk war der bevorzugte Style der Hipster und Hustler, seine Träger galten nicht selten als sexy und gefährlich, wie eine Afroamerikanerin im Interview mit Craig deutlich macht: „I liked men with conks. They were grand. They were the other side“. ${ }^{40}$ Zum anderen unterschied sich der Conk der Zoot Suiters und Hipsters sehr von dem der afroamerikanischen Bourgeoisie, der kürzer und weniger flamboyant getragen wurde und somit eher einem konventionellen und als wei $\beta$ codierten Herrenhaarschnitt glich. ${ }^{41}$ In der Tat widersetzte sich der Conk jeglichen Vorstellungen der Schwarzen Mittelschicht und der weißen Mehrheit davon, wie ein Schwarzer respektabler Mann auszusehen hatte. Im Gegensatz zum geglätteten Haar Schwarzer Frauen, was weder als verrucht noch als unanständig galt, sondern vielmehr einen disziplinierten Körper implizierte, der sich modern, respektabel und fortschrittlich gab, betonte der Conk Differenz:

37 Malcolm X: The Autobiography of Malcolm X. With the Assistance of Alex Haley. New York: Ballantine Books 1965, S. 54.

38 Vgl. Ebd., S. 52-55; siehe auch B. Tyler: Black Hairstyles.

39 Zitiert nach M. Craig: Ain't I a Beauty Queen?, S. 408.

40 Zitiert nach Ebd., S. 399.

41 Vgl. R. Kelley: Nap Time, S. $167 f$. 
No, the conk did not copy anything, and certainly not any of the prevailing white male hairstyles of the day. Rather, the element of straightening suggested resemblance to white people's hair, but the nuances, inflections and accentuations introduced by artificial means of stylization emphasized difference. In this way the political economy of the conk rested on its ambiguity, the way it played with the given outline shapes of convention only to disturb the norm and hence invite a ,double take' demanding that you look twice. ${ }^{42}$

In seinen verschiedenen Variationen kann der Conk vielmehr als Parodie zum einen auf weißes Haar und zum anderen auf die Versuche der Schwarzen Mittelschicht, „gutes“/glattes Haar zu bekommen, gedeutet werden. Gerade junge Männer wie Malcolm X, die einen Zoot Suit trugen, brachen mit der dominanten Männermode der Zeit, da der verschwenderisch geschnittene und oft in grellen Farben getragene Zoot Suit/Anzug breite Schultern und schmale Hüften ins Lächerliche zog. Diese Performanz als verzweifelten Versuch, weiß-sein zu wollen zu interpretieren, geht an der Komplexität und Vielschichtigkeit der Inszenierung vorbei. Anhand dieses Beispiels wird vielmehr deutlich, dass es bei dem Conk nicht um die Imitation einer euroamerikanischen Ästhetik geht, sondern dass es sich bei dieser Schwarzen Ästhetik um die Artikulation verschiedener Variablen handelt, die sich in den Körperpraktiken manifestieren:

Within black communities before 1965, hair signified as much about class and gender as it did about race. (...) some black men who were positioned as ,other' because they were black and who were again positioned as ,other' by their exclusion from middle-class occupations claimed the position of ,other and from that location created a black and masculine style: the conk. 43

Im Gegensatz zu Afroamerikanerinnen, die ihre Haare glätteten und sich damit innerhalb der gängigen Geschlechter- und Mittelklassekonventionen bewegten, galt das geglättete, das geconkte Haar von jungen Afroamerikanern als ausdrücklich nonkonform und deviant. Es trotzte jedem bürgerlichen Anspruch und fühlte sich sicherlich nicht dem „racial uplift“ verpflichtet, wie die Sorge und Pflege um sich selbst und das eigene Äußere zuvor noch von Madame C. J. Walker oder Booker T. Washington propagiert worden waren. Die Art und Weise, wie Malcolm X seinen Conk rückblickend als einen verzweifelten, traurigen Ausdruck von Selbsthass und krankem Wunsch nach weiß-sein beschreibt, veranschaulicht exemplarisch den politischen Wandel innerhalb der afroamerikani-

42 K. Mercer: Welcome to the Jungle, S. 119 (Hervorhebung im Original).

43 M. Craig: Ain't I a Beauty Queen?, S. 402f. 
schen Communities von den 1940ern bis zu den 1960ern. Während der späten 1960er Jahre wurde der Conk von etlichen Schwarzen, afrozentrischen und Schwarz-nationalistischen Aktivisten wie Maulana Karenga, Stokeley Carmichael, oder eben Malcolm X kritisiert. Sie sahen den Conk nicht als subversive, ästhetische Inszenierung des Subjekts, das sich von ethischen Konventionen befreite und eine alternative Ästhetik propagierte. Aufgrund des Elements des straightening, dass dem Conk innewohnt, konnte er in diesem politischen Klima nur als Internalisierung einer weißen, unterdrückenden Ästhetik und Politik gedeutet werden.

Aufgrund der Tatsache, dass die afroamerikanische Bürgerrechtsbewegung ab den 1950er Jahren eine nationale und internationale Dynamik erhalten und an Stoßkraft gewonnen hatte, wurde das Schwarze Selbstbild einer verstärkten Analyse unterzogen, die oftmals darin mündete, dass das eigene Aussehen stärker politisiert, beziehungsweise mit konkreten Black Power-Ideologien in $\mathrm{Zu}$ sammenhang gebracht wurde. Es vollzog sich ein Wandel, der das Monopol des hair straightening zwar nicht aufhob, aber dennoch einen alternativen Style offerierte, der von Vielen aus mitunter unterschiedlichen Motiven aufgegriffen wurde.

Im August 1963 erschien ein Artikel in der Negro Digest, der mit der Frage betitelt war „Should Negro women straighten their hair?“ Durchaus gegensätzliche Antworten gaben eine afroamerikanische Mitherausgeberin des Hochglanzmagazins Jet, Helen Hayes King, und eine nigerianische Journalistin für die Times, Theresa Ogunbiyi. Ogunbiyi wehrt sich gegen männliche Kritiker aus dem eigenen Land, die ihr vorwerfen, sie propagiere europäische Mode und Lifestyle, anstatt die Vielfalt an nigerianischen Frisuren und „traditionellen“ Bekleidungen zu zelebrieren. In ihrer Verteidigung von Glätteisen wiederholt sie die vorherrschende Meinung der ersten Hälfte des 20. Jahrhunderts, indem sie geglättetes Haar mit Fortschritt und Modernität gleich setzt. Geglättetes Haar war für Ogunbiyi untrennbar mit einer modernen und fortschrittlichen nigerianischen Gesellschaft verbunden, so wie diese Haarpraxis für Madame C. J. Walker mit der Fortschrittlichkeit Schwarzer Frauen verknüpft war. Die Afroamerikanerin Helen Hayes King vertrat wiederum die Ansicht, der „au naturelle“-Look, wie die Spielarten des Afro auch genannt wurden, praktikabler und besser für ihr Haar seien. Sie berichtet von Jahren der Frustration, weil das Schönheitsideal von langen, glatten Haaren für sie unerreichbar war, und vom ersten Besuch im Schönheitssalon, wo das straighhtening nicht dem erhofften Initiationsritus glich, dem Übergang von einem jungen Mädchen zu einer erwachsenen Frau, wie es oft beschrieben wird, sondern einen Moment von Enttäuschung und Verrat darstellte. Auch wenn King argumentiert, dass „I'm not so involved in the neo- 
African aspects of the „au naturelle“ look, nor in the get-back-toyour-heritage bin, (...). All I know is that me and my ,baby fine locks never looked or felt better!"44; die Tatsache, dass sie als prominente Vertreterinnen des „natürlichen“ Styles Frauen wie Miriam Makeba, Odetta oder Abbey Lincoln, -alles politische Frauen, die sowohl den Black Freddom Struggle als auch die afrikanischen Unabhängigkeitsbewegungen offen unterstützten- zitiert, legen den Schluss nahe, dass der Afro politische Konnotationen hatte und nicht nur ein New Yorker Modetrend war, beziehungsweise bleiben sollte.

\section{„Kinky Hair - the Badge of Rebellion“}

Da der Afro oft als „natürlicher“ Style bezeichnet wurde, ist es nicht unwichtig, sich vor Augen zu führen, dass es eine „natürliche“ Haarfrisur als solche nicht geben kann. Haar ist niemals „a straightforward biological fact, because it is almost always groomed, prepared, cut, concealed and generally worked upon by human hands. (...) In this way hair is merely a raw material, constantly processed by cultural practices which thus invest it with meanings and value“.45 Diskurse um „natürliches“ Haar laufen darüber hinaus Gefahr, körperliche Charakteristika wie Hautfarbe und Haartextur zu naturalisieren, wodurch die kulturellen Praktiken und Strategien, um die es eigentlich geht, verschleiert werden. Bei der Referenz auf „natürliches“ Haar und somit auf eine afrikanische Herkunft handelt es sich indes um ein wichtiges politisches Argument, dass im Zuge der Black is Beautiful-, und der Black PowerBewegungen immer lauter wurde und in dessen Kontext Zuschreibungen wie „Black“ und „natural hair“ zurück gefordert und positiv konnotiert worden sind. Innerhalb dieses Diskurses fungiert ein „natürlicher“ Style auch als Ausdruck eines politischen Bewusstseins und einer afrozentrischen Politik. ${ }^{46}$

44 Helen Hayes King/Theresa Ogunbiyi: „Should Negro Women Straighten Their Hair?", in: Negro Digest (April 1963), S. 65-71, hier S. 71.

45 K. Mercer: Welcome to the Jungle, S. $100 f$.

46 Obwohl politisch ein wichtiges Argument, kritisiert Cornel West Afrozentrismus als zu kurz gedachte Kritik: „Afrocentrism, a contemporary species of black nationalism, is a gallant yet misguided attempt to define an African identity in a white society perceived to be hostile. It is gallant because it puts black doings and sufferings, not white anxieties and fears, at the center of discussion. It is misguided because - out of fear of cultural hybridization and through silence on the issue of class, retrograde views on black women, gay men, and lesbians, and a reluctance to link race to 
Der Afro war vor allem in urbanen Zentren, unter Studierenden, Künstlern und Intellektuellen zu finden, zog sich jedoch nicht quer durch alle afroamerikanischen Communities. Sein Charakteristikum war die Länge. Mit Hilfe eines grobzackigen Kammes, dem Afro comb, oder Afro pick, wurde das Haar dazu gebracht, nach oben und nach außen zu wachsen und es ergab sich die typisch runde Form dieses Styles. Der Afro kommunizierte Schwarzen Stolz, was fast schon zu einem Synonym für Schwarzen Nationalismus wurde, nachdem er -kombiniert mit schwarzem Berret, schwarzer Lederjacke und, bei Bedarf, schwarzer Schrotflinte- zum erklärten Look der Black Panther Party geworden war. In New Day in Babylon konstatiert William Van DeBurg, dass die Black Power-Bewegung eine Revolution der Schwarzen Kultur initiierte. Soul wurde zu einem der wichtigsten Schlagwörter und der Inbegriff dessen, wie eine andere, neue Blackness imaginiert wurde. Es gab nicht nur Soul Music und Soul Food, sondern auch einen Soul Style, dessen unabdingliches Accessoire ein Afro war. ${ }^{47}$ In Anlehnung an den eingangs zitierten Stokeley Carmichael und an Malcolm X analysiert Van DeBurg den Afro als Ausdruck eines gesunden, selbstbewussten Schwarz-seins und hair straightening als einen Versuch der Assimilation an euroamerikanische Schönheits-standards. Die Tatsache, dass der Afro zu dem Symbol der Black Power-Bewegung geworden ist und in diesem Diskurs Eingang in die Populärkultur gefunden hat, macht es nach Kelley „impossible for many contemporary writers to see beyond the raised-fisted militant attired in black turtlenecks or faux African garb“.48 Tatsächlich aber, wie oben bereits angedeutet, erlangte der Afro eine gewisse Prominenz unter dem Namen „au naturelle“ oder „au natural“ in der euro- und afroamerikanischen New Yorker Bourgeoisie der späten 1950er Jahre. Junge Afroamerikanerinnen und Euroamerikanerinnen, die sich in avantgardistischen und kulturell diversen Kreisen in Manhatten bewegten, lernten den Afro, auch „the Natural“ genannt, durch Models kennen, die ihn bei sogenannten „au naturel shows“ trugen, die damals der neueste Trend waren. ${ }^{49}$ Der Style galt als feminin, als „exotisch“ und extravagant. Manche Frauen bevorzugten ihn, um ihr Haar nicht mehr mit chemischen Produkten und Glätteisen malträtieren zu müssen, für andere war er Ausdruck von Solidarität mit den neuen, unabhängigen Nationen Afrikas. Maya Angelou, Miriam Makeba, Nina Simone und Abbey Lincoln gehörten alle zu den frühen Vertreterinnen des „natural look“, und da sie alle Verfechte-

the common good - it reinforces the narrow discussion about race" (Cornel West: Race Matters. Boston, MA: Beacon Press 1993, S. 4).

47 Vgl. W. Van Deburg: New Day in Babylon, S. $197 \mathrm{f}$.

48 R. Kelley: Nap Time, S. 340.

49 Vgl. Ebd., S. 341. 
rinnen der afrikanischen Unabhängigkeitsbestrebungen und der afroamerikanischen Bürgerrechtsbewegung waren, verwundert es nicht, dass ihr Style mit einer bestimmten Politik verknüpft worden ist. Er befand sich außerdem im Einklang mit der politisch wichtigen und einflussreichen Black is Beautiful-Bewegung, die die Betonung und Wertschätzung von distinkten afrikanischen Charakteristika wie der Haartextur beinhaltete.

Es wird jedoch auch deutlich, dass der Afro von den ausgehenden 1950er bis zu den frühen 1970er Jahren mit verschiedenen, auch gegensätzlichen Konnotationen versehen war, bevor er fest in einem maskulin geprägten Black Power-Diskurs verankert wurde. Zum einen entstand der Afro als ein schickes Accessoire der Bourgeoisie; er wurde erst ab Mitte der 1960er Jahre zu einem populären Symbol für afroamerikanische Rebellion. Zum anderen wurde der Afro, in Anlehnung an seine Trägerinnen bei den „au naturel shows“, zunächst als femininer Haarschnitt konstruiert. Er hatte gerade für Afroamerikanerinnen eine besondere Bedeutung, weil er das betonte, was an ihren Haaren distinkt Schwarz war, und weil es keiner großen Operationen bedurfte, das Haar zum Afro zu tragen (vorausgesetzt, dass Haar war stark genug gelockt). Diese Konnotation des Afro als weiblicher chic $^{50}$ rückte im Laufe der Zeit jedoch mehr und mehr in den Hintergrund, und er wurde das „symbol of black manhood, the death of the "Negro' and the birth of the militant, virulent Black man“. ${ }^{51}$ Eine Konsequenz dieser Entwicklung war, dass Frauen, die den Afro weiterhin trugen, nachdem er diskursiv als männlich und militant konstruiert worden war, als weniger weiblich erachtet wurden, beziehungsweise sich vorwerfen lassen mussten, offensichtliche Geschlechtergrenzen und -normen zu überschreiten. ${ }^{52}$ Von seinen Trägerinnen wurde der Afro daher explizit in Verbindung gebracht mit Unterdrückungen entlang der Variablen race und gender. Er stellte eine Rückgewinnung der eigenen Schwarzen Körperlichkeit ebenso dar wie er einen Bruch mit den gängigen Vorstellungen von Femininität deklarierte. ${ }^{53}$ In diesem $\mathrm{Zu}$ sammenhang fungierte der Afro nicht nur als eine Gegentechnologie

$50 \mathrm{Vgl}$. Lois Liberty Jones /John Henry Jones: All About the Natural. Clairol, 1971; das Buch wurde von dem Kosmetikkonzern Clairol produziert, und bietet Frauen, neben einer kurzen historischen Einführung in Haarpraktiken und deren politische Bedeutung, Tipps zur Pflege eines Afros mit Clairol-Produkten, sowie bildhafte Anregungen für verschiedene feminine AfroFrisuren mit erstaunlich klingenden Namen wie "Miss Zanzie“, „Soul Love“, oder „Freedom Burst" (Schomburg Center for Research in Black Culture, New York Public Library, New York).

51 Ebd., S. 344.

52 Vgl. M. Craig: Ain't I a Beauty Queen?, S. 125.

53 Vgl. S. Walker: Style and Status, S. $179 f$. 
zur dominanten rassistischen Gesellschaft, die euroamerikanische Schönheitsstandards diktierte, sondern auch als ein Gegenentwurf zum Sexismus innerhalb der afroamerika-nischen Communities und deren mehrheitliche Auffassungen darüber, wie eine respektable, Schwarze Frau auszusehen hatte.

Als junge Studentinnen Mitte der Sechziger Jahre begannen, Afros an ihren Universitäten zu tragen, wurde dies von den KommilitonInnen und ProfessorInnen nicht ausschließlich begeistert aufgenommen. Zohara Simmons, Mitglied des Student Nonviolent Coordinating Committee (SNCC) erinnert sich, als sie zum ersten Mal einen Afro am Spelman College in Atlanta, Georgia trug:

I began wearing an Afro my second year at Spelman, and it caused a big furor. I was actually called into the dean's office, and she asked me ,What happened to your hair?' I said, ,l'm wearing an Afro.' And she (said), ,Well that's not how a Spelman young woman is supposed to look. You have to be well groomed.' I said, ,I am well groomed. I just happen not to have my hair straightened'.54

Offensichtlich war die Rektorin nicht einverstanden mit der neuen Frisur Simmons', weil diese kulturelle Normen von Weiblichkeit und Schwarzem Mittelklasse-Verhalten verletzte. 1966 kandidierte die Studentin Robin Gregory um den Titel der homecoming queen an der traditionell Schwarzen Howard University in Washington, D.C., nachdem sie von Freunden gefragt worden war: „Well, will you do this? We want to run somebody that has a natural hairstyle. We know that you're politically active. Let's take this particular context and use it to make a statement".55 Gregory verzichtete auf aufwändige Kleider und große, inszenierte Auftritte wie es ihre Mitbewerberinnen taten. Stattdessen gestaltete sie ihre Kampagne explizit politisch und hielt Reden über die Position von Frauen in der afroamerikanischen Bürgerrechtsbewegung, was die Professorin Paula Giddings nachhaltig beeindruckte: „And what Robin did was not only in terms of race, but also talking about the role of women and what they should be doing and talking about and being taken very, very seriously, not just because of any physical attributes but because of her mind“. Zur großen Überraschung wurde Gregory zur homecoming queen gewählt, der ersten, die einen Afro trug: „Well, before you saw Robin, you saw the way the lights cast a silhouette on the curtains, and you saw the silhouette of her Afro before you saw her. Well, the auditorium exploded. It was a wonderful mo-

54 Zitiert nach M. Craig: Ain't I a Beauty Queen?, S. 134.

55 Henry Hampton/Steve Fayer: Voices of Freedom. An Oral History of the Civil Rights Movement from the 1950s through the 1980s. New York, Bantam Books 1990, S. 433 f. 
ment“. ${ }^{56}$ Am Ende der Sechziger Jahre war der Afro an sämtlichen Universitäten in städtischen Gebieten weit verbreitet und wurde von vielen jungen AfroamerikanerInnen als Ausdruck ihrer Identifikation mit dem Black Freedom Struggle getragen.

Das Tragen eines 'Fros entwickelte sich zusehends zu einem „Muss“ für politisch bewusste AfroamerikanerInnen und zu einer moralischen Verpflichtung. Aufgrund dieses policing of hair, wie es von einigen empfunden wurde, weigerte sich beispielsweise Carolivia Herron, Autorin des Kinderbuches Nappy Hair, während ihrer Zeit als Studentin an der Howard University, einen Afro zu tragen. Rückblickend erklärt sie dies folgendermaßen: „When I was at Howard and everyone was going natural with Afros, I refused to do it because I wanted to show that I could be just as radical as they were without being it in my hair. Wearing an Afro didn't mean you had your soul in the right place“. ${ }^{57}$ Herron wehrte sich gegen die komplette Verschmelzung von Politik und Ästhetik und betont, dass eine Haarfrisur nicht als determinierende Zuschreibung für die eigene politische Haltung gelesen werden darf. Eine tendenziell gegensätzliche Ansicht vertritt Angela Davis, die durch ihr FBIFahndungsfoto, auf dem sie einen riesigen Afro trägt, nicht nur zweifelhafte internationale Berühmtheit erlangte, sondern auch zu einer Ikone der Black Power-Bewegung wurde (Abb. 2).

56 Ebd., S. 435.

57 Zitiert nach A. Byrd/L. Tharps: Hair Story, S. 66; Carolivia Herron ist die Autorin des Kinderbuches Nappy Hair, das Ende 1998 einen nationalen Skandal auslöste, nachdem es von einer weißen Lehrerin im Unterricht verwendet worden war. Die Grundschüler, mehrheitlich Schwarz und Latino, waren so begeistert von dem Buch, dass die Lehrerin Fotokopien von der Hauptperson des Buches, der kleinen Brenda, auf denen sie mit einem riesigen Afro zu sehen war, anfertigte. Diese Kopien jedoch wurden von einigen Eltern als rassistisch empfunden. Das Ganze mündete in einem Eklat, der zur Versetzung der Lehrerin führte; nationale Medien berichteten über den Vorfall. Jill Nelson vertrat den Standpunkt, dass das Problem innerhalb der afroamerikanischen community bestand, und dass die Lehrerin unwissentlich über "the absurdity of a race secret" gestolpert sei, indem sie sich nicht der problematischen Beziehung bewusst gewesen sei, die manche AfroamerikanerInnen zu ihrem eigenen Haar haben (Vgl. Carolivia Herron: Nappy Hair. New York: Knopf 1997; Jill Nelson: "Stumbling Upon a Race Secret", in: The New York Times, 27.11.1998; Michelle H. Martin „Never Too Nappy“, in: The Horn Book Magazine 75 (1999), S. 283-288. 


\section{Angela Davis trifft Erich Honecker}

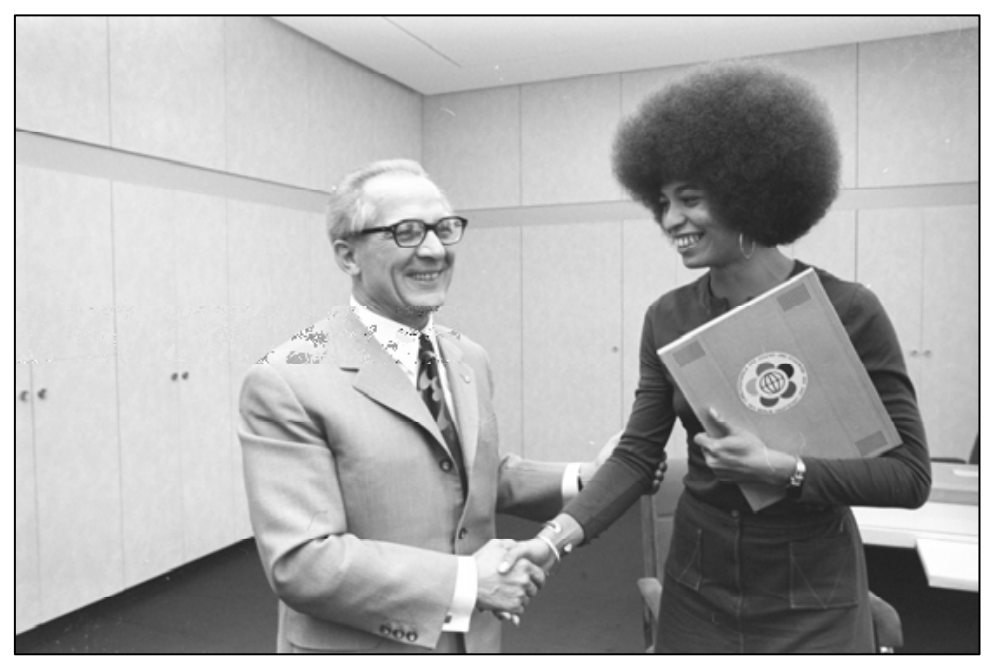

Abb. 2: Angela Davis mit ihrem Trademark-Afro, der sie national und international zu einer Ikone der Black Power-Bewegung werden ließ, bei einem Empfang bei Erich Honecker in Berlin. „Der Erste Sekretär des ZK der SED, Erich Honecker, empfing am 11.9.72 die

amerikanische Bürgerrechtskämpferin Angela Davis. Während dieser Begegnung überreichte er der Vertreterin des anderen Amerikas die Einladung für die Weltfestspiele der Jugend und Studenten 1973 in der DDR-Hauptstadt. “

Davis, eine der prominentesten Frauen in der Black Panther Party, deren inhärenten Sexismus sie immer wieder anprangerte, betont, wie wichtig für sie persönlich die Verknüpfung von Schwarznationalistischer Politik und Schwarzer Ästhetik war: „I needed to say ,Black is beautiful' as much as any of the intransigent antiwhite nationalists. I needed to explore my African ancestry, to don African garb, and to wear my hair natural as much as the blinderwearing male supremacist cultural nationalists“.58 Der Afro als Gegentechnologie ist hier untrennbar verbunden mit der Entwicklung eines bestimmten politischen Bewusstseins, zu dem der Rückbezug auf Afrika ebenso gehört wie die Rückgewinnung der eigenen Schwarzen Körperlichkeit. Kritisch betrachtet Davis allerdings die

58 Angela Davis: „Black Nationalism: The Sixties and the Nineties“, in: Joy James (Hg.), The Angela Y. Davis Reader. Malden, MA: Blackwell Publishers 1998, S. $319 f$. 
nachträgliche, limitierende Kontextualisierung des Afros in einem Nationalismus, der sexistisch und misogyn war, und gegen den sie gekämpft hat.

Die afroamerikanische Schönheitsindustrie war nicht ausschließlich enthusiastisch, was den Afro betraf. Skeptiker befürchteten, dass der Style, entgegen seiner politischen Konnotation, den afroamerikanischen Communities schaden würde, da den Friseursalons die Kundschaft ausblieb, wenn Frauen und Männer ihre Haare „natürlich“ wachsen ließen.59 In der Tat war es ein wirtschaftlicher Nebeneffekt der Popularität des Afro, dass etliche Friseursalons bankrott gingen oder mit schweren finanziellen Einbußen zu kämpfen hatten, da die, die einen Afro favorisierten, nicht mehr wöchentlich ihr Haar glätten und frisieren ließen, sondern höchstens alle paar Wochen einen Salon aufsuchten, um den Afro „rund“ schneiden zu lassen. Dies taten junge Frauen dann meist auch in den barbershops, wodurch der Afro eine zusätzliche Maskulinisierung erfuhr, und nicht in den beauty parlors, wo er oft abfällige Bemerkungen provozierte. ${ }^{60} \mathrm{Um}$ die negativen Konsequenzen im Zaum zu halten und um das Vermarktungs- und Verkaufspotential des Afros bestmöglich zu nutzen, überfluteten gleichwohl Haarpflegeprodukte den Markt, die sich einer Black Pride-Rhetorik bedienten, beispielsweise Hill's Egyptian Oil Co. oder Black Magic Afro Grow. Eine weitere ökonomische Nische waren Afro-Perücken für diejenigen, deren eigenes Haar das Tragen eines Afros nicht zulie $\beta$, oder die sich nicht vollständig auf diesen „revolutionären Look“ festlegen wollten. ${ }^{61}$ Im Editorial der Branchenzeitschrift Beauty Trade vom Januar 1972 forderte Chefredeakteurin Willa Lee Calvin ihre Leserinnen und Leser auf: „If, in this still young and bright 1972, you remember just one thing: Quit blaming Afros for bad business. The Naturals are here to stay -too many think they are fashionably beautiful when cut and styled becomingly. You might as well get the conditioning business they automatically generate as well as do Afros“. 62

Zum Ende der 1970er Jahre war der Afro zu einer Frisur unter vielen geworden, die nicht mehr unmittelbar in einem Black PowerKontext gelesen wurde, obgleich sie den Weg für die Verbreitung und Popularisierung anderer, afrikanisch-inspirierter Styles wie

59 Vgl. S. Walker: Style and Status, S. 189.

60 Vgl. M. Craig: Ain't I a Beauty Queen?, S. 124.

61 Vgl. A. Byrd/L. Tharps: Hair Story, S. 63; Vertamae Smart-Grosvenor: „Heady Times“, in: American Visions 14 (1999), S. 16-22; Susannah Walker: „Black is Profitable: The Commodification of the Afro, 1960-1975“, in: Enterprise \& Society 1 (2000), S. 536-547.

62 Willa Lee Calvin: „Memo from the Editor's Desk“, in: Beauty Trade Magazine 18 (Januar 1972), S. 6 
Cornrows, Braids und Dredlocks eröffnete. Somit war der Afro als Widerstandsmoment aufgrund der Instabilität der Mode nur temporär wirksam. Ein distinkter Hairstyle mag helfen, neue „radikale“ Ideen zu kommunizieren und zu verbreiten, er kreiert ein Gruppengefühl unter denen, die sich mit der Bewegung und dem Style identifizieren. Eine nachhaltige Bedeutung, beziehungsweise eine langfristige Wirksamkeit ist jedoch schwierig auszumachen, da unausweichlich eine gewisse „Verwässerung“ eintritt, sobald der Afro von Vielen getragen wird. Je mehr Menschen einen Style adaptieren, desto wahrscheinlicher ist es, dass er zum einen an Radikalität einbüßt, und zum anderen kommodifiziert und in den modischen Mainstream überführt wird, quasi als Konsum ethnischer Diversität, beziehungsweise sich zu einem bloßen Modeereignis zurück entwickelt.

Gerade weil Schwarze Körper den Disziplinierungsmaßnahmen einer dominanten weißen Gesellschaft stärker ausgesetzt sind, ist der Körper auch ein Ort für Widerstand. Dieser kann sich in vermeintlich individuellen Entscheidungen wie der Haarfrisur manifestieren, die jedoch auch immer zumindest die Möglichkeit einer explizit politischen Komponente implizieren. Eine Reduzierung auf eine rein politische Ebene, die eine ästhetische und ethische Inszenierung unberücksichtigt lässt, ist jedoch zu einseitig gedacht, denn, so Lola Young:

There are, in truth, a multiplicity of reasons for doing what we do to our bodies, our skin, our hair, some of which may not be as easily containable in terms like ,black consciousness ' or ,natural' or ,coconut' as those who wish to regulate us would like to think. The act of looking for signifiers of political consciousness, racial integrity and authenticity is an attempt to control, to ,master', in order to be able continually to reaffirm the coherence and stability of the self. 63

Dieser Wunsch, beziehungsweise diese Suche nach Signifikanten in afroamerikanischen Haarpraktiken sowie die Debatten, die innerhalb der afroamerikanischen Bevölkerung geführt worden sind, wurden hier anhand ausgewählter populärer Styles im 20. Jahrhunderts diskutiert. Die Diskurse um straightened Hairstyles, um den Conk und den Afro verdeutlichen, dass Schwarzes Haar als ein umkämpfter Ort fungiert, der durch Auffassungen von race, class und gender determiniert wird, und dass die Diskurse um Haar in verschiedenen Technologien des Selbst münden. Diese Technologien verlaufen nicht notwendigerweise konform mit gesellschaftlichen

63 Lola Young: „How Do We Look? Unfixing the Singular Black (Female) Subject", in: Paul Gilroy/Lawrence Grossberg/Angela McRobbie (Hg.), Without Guarantees. In Honour of Stuart Hall. London: Verso 2000, S. $426 \mathrm{f}$. 
Erwartungen, sondern lassen dem Individuum Raum für widerständige Positionierungen durch eine Vielzahl von Körperpraktiken.

\section{Literatur}

Battle-Walters, Kimberly: Sheila's Shop. Working-Class African American Women Talk About Life, Love, Race, and Hair. Lanham, MD: Rowman \& Littlefield 2004.

Blackwelder, Julia K.: Styling Jim Crow. African American Beauty Training During Segregation. College Station, TX: Texas A\&M University Press 2003.

Bordo, Susan: Unbearable Weight. Feminism, Western Culture, and the Body. Berkeley, CA: University of California Press 2003.

Bundles, A'Lelia: On Her Own Ground. The Life and Times of Madam C. J. Walker. New York: Scribner 2001.

Butler, Judith: Bodies that Matter. On the Discursive Limits of „Sex.“ London, New York: Routledge 1993.

Byrd, Ayana/Tharps, Lori L.: Hair Story. Untangling the Roots of Black Hair in America. New York: St. Martin's Press 2001.

Craig, Maxine: Ain't I a Beauty Queen? Black Women, Beauty, and the Politics of Race. Oxford, New York: Oxford University Press 2002.

Davis, Angela: „Black Nationalism: The Sixties and the Nineties“, in: Joy James (Hg.), The Angela Y. Davis Reader. Malden, MA: Blackwell Publishers 1998.

DuCille, Ann: Skin Trade. Cambridge, MA: Harvard University Press 1996.

Foucault, Michel: „Die Ethik der Sorge um sich als Praxis der Freiheit“ (1984), in: Ebd., Ästhetik der Existenz. Schriften zur Lebenskunst. Frankfurt/Main: Suhrkamp Verlag 2007.

Ders.: „Technologien des Selbst“ (1982), in: Ebd., Ästhetik der Existenz. Schriften zur Lebenskunst. Frankfurt/Main: Suhrkamp Verlag 2007.

Gates, Henry L., Jr.: „It all Comes Down to the Kitchen“, in: Juliette Harris/Pamela Johnson (Hg.), Tenderheaded. A Comb-Bending Collection of Hair Stories. New York: Pocket Books 2001.

Hampton, Henry/Fayer, Steve: Voices of Freedom. An Oral History of the Civil Rights Movement from the 1950s through the 1980s. New York, Bantam Books 1990.

Herron, Carolivia: Nappy Hair. New York: Knopf 1997.

Higbee, Mark: „The Hairdresser and the Scholar“, in: Juliette Harris/Pamela Johnnson (Hg.), Tenderheaded. A Comb-Bending Collection of Hair Stories. New York: Pocket Books 2001. 
hooks, bell: „Straightening Our Hair“, in: Juliette Harris/Pamela Johnson (Hg.), Tenderheaded. A Comb-Bending Collection of Hair Stories. New York: Pocket Books 2001.

Kaba, Mariame: „When Black Hair Tangles With White Power“, in: Juliette Harris/Pamela Johnson (Hg.), Tenderheaded. A CombBending Collection of Hair Stories. New York: Pocket Books 2001.

Kelley, Robin D. G.: „Nap Time: Historicizing the Afro“, in: Fashion Theory 1 (1997), S. 339-352.

Ders.: Race Rebels. Culture, Politics, and the Black Working Class. New York: The Free Press 1994.

King, Helen Hayes/Theresa Ogunbiyi: „Should Negro Women Straighten Their Hair?“, in: Negro Digest (April 1963), S. 65-71.

Lake, Obiagele: Blue Veins and Kinky Hair. Naming and Color Consciousness in African America. Westport, CT: Praeger 2003.

Lee Calvin, Willa: „Memo from the Editor's Desk“, in: Beauty Trade Magazine 18 (Januar 1972), S. 6.

Lewis, David Levering : W.E.B. Du Bois. The Fight for Equality and the American Century, 1919-1963. New York: Henry Holt 2000.

Malcolm X: The Autobiography of Malcolm X. With the Assistance of Alex Haley. New York: Ballantine Books 1965.

Martin, Michelle H.: „Never Too Nappy“, in: The Horn Book Magazine 75 (1999), S. 283-288.

Mercer, Kobena: Welcome to the Jungle. New Positions in Black Cultural Studies. London, New York: Routledge 1994.

Nelson, Jill: „Stumbling Upon a Race Secret“, in: The New York Times, 27.11.1998.

Patton, Tracy O.: „Hey Girl, Am I More than my Hair? African American Women and their Struggles with Beauty, Body Image and Hair“, in: National Women's Studies Journal 18 (2006), S. 24-51.

Rooks, Noliwe M.: Ladies' Pages. African American Women's Magazines and the Culture that Made them. New Brunswick, NJ: Rutgers University Press 2004.

Dies.: Hair Raising. Beauty, Culture, and African American Women. Piscataway, NJ: Rutgers University Press 1996.

Smart-Grosvenor, Vertamae: „Heady Times“, in: American Visions 14 (1999), S. 16-22.

Tyler, Bruce M.: „Black Hairstyles. Cultural and Socio-Political Implications“, in: The Western Journal of Black Studies 14 (1990), S. 235-250.

Van Deburg, William L.: New Day in Babylon. The Black Power Movement and American Culture, 1965-1975. Chicago, IL: University of Chicago Press 1992. 
Walker, Susannah: Style and Status. Selling Beauty to African American Women, 1920-1975. Lexington, KY: The University Press of Kentucky 2007.

Dies.: „Black is Profitable: The Commodification of the Afro, 19601975“, in: Enterprise \& Society 1 (2000), S. 536-547.

Weitz, Rose: „Women and their Hair. Seeking Power through Resistance and Accommodation“, in: Gender \& Society 15 (2001), S. 667-86.

Young, Lola: „How Do We Look? Unfixing the Singular Black (Female) Subject“, in: Paul Gilroy/Lawrence Grossberg/Angela McRobbie (Hg.), Without Guarantees. In Honour of Stuart Hall. London: Verso 2000.

\section{ABBILDUNGSNACHWEISE}

Abb. 1 aus: African American Cosmetic Labels Collection, Archives Center, National Museum of American History, Behring Center, Smithsonian Institution, Washington, D.C.

Abb. 2 aus: Bundesarchiv. 
